PROCEEDINGS OF THE

AMERICAN MATHEMATICAL SOCIETY

Volume 128, Number 3, Pages 775-780

$\mathrm{S}$ 0002-9939(99)05600-2

Article electronically published on October 20, 1999

\title{
ON $p$-HYPONORMAL OPERATORS
}

\author{
EUNGIL KO
}

(Communicated by David R. Larson)

\begin{abstract}
In this paper we show that $p$-hyponormal operators with $0 \notin$ $\sigma\left(|T|_{r}^{\frac{1}{2}}\right)$ are subscalar. As a corollary, we get that such operators with rich spectra have non-trivial invariant subspaces.
\end{abstract}

\section{INTRODUCTION}

Let $H$ and $K$ be separable complex Hilbert spaces and let $\mathcal{L}(H, K)$ denote the space of all bounded linear operators from $H$ to $K$. If $H=K$, we write $\mathcal{L}(H)$ in place of $\mathcal{L}(H, K)$.

An operator $T \in \mathcal{L}(H)$ is said to be $p$-hyponormal, $0<p \leq 1$, if $\left(T^{*} T\right)^{p} \geq$ $\left(T T^{*}\right)^{p}$ where $T^{*}$ is the adjoint of $T$. If $p=1, T$ is called hyponormal and if $p=\frac{1}{2}$, $T$ is called semi-hyponormal. Semi-hyponormal operators were introduced by Xia (see $[\mathrm{Xi}]$ ), and $p$-hyponormal operators for a general $p, 0<p<1$, have been studied by Aluthge. Any $p$-hyponormal operators are $q$-hyponormal if $q \leq p$. But there are examples to show that the converse of the above statement is not true (see [Al]).

A bounded linear operator $S$ on $H$ is called scalar of order $m$ if it has a spectral distribution of order $m$, i.e., if there is a continuous unital morphism of topological algebras

$$
\Phi: C_{0}^{m}(\mathbf{C}) \longrightarrow \mathcal{L}(H)
$$

such that $\Phi(z)=S$, where as usual $z$ stands for the identity function on $\mathbf{C}$ and $C_{0}^{m}(\mathbf{C})$ stands for the space of compactly supported functions on $\mathbf{C}$, continuously differentiable of order $m, 0 \leq m \leq \infty$. An operator is subscalar if it is similar to the restriction of a scalar operator to a closed invariant subspace. We now define the weaker form of a subscalar operator. An operator $T \in \mathcal{L}(H)$ is quasi-subscalar if there exists a one-to-one $V \in \mathcal{L}(H, K)$ such that $V T=S V$ where $S(=\Phi(z)$ in the above definition) is a scalar operator.

This paper has been divided into three sections. Section 2 deals with some preliminary facts. In section 3 , we show that $p$-hyponormal operators with the property $0 \notin \sigma\left(|T|_{r}^{\frac{1}{2}}\right)$ are subscalar. As a corollary, we get that such operators with rich spectra have non-trivial invariant subspaces.

Received by the editors April 22, 1998

2000 Mathematics Subject Classification. Primary 47B20, 47A15.

Key words and phrases. p-hyponormal, subscalar operators, invariant subspaces.

The author is supported by the MOST through National R \& D Program (97-N6-01-01-A-5) for Women's Universities.

(C)1999 American Mathematical Society 


\section{Preliminaries}

Let $d \mu(z)$, or simply $d \mu$, denote the planar Lebesgue measure. Let $H$ be a complex separable Hilbert space, and let $D$ be a bounded open disc in $\mathbf{C}$. We shall denote by $L^{2}(D, H)$ the Hilbert space of measurable functions $f: D \rightarrow H$, such that

$$
\|f\|_{2, D}=\left(\int_{D}\|f(z)\|^{2} d \mu(z)\right)^{\frac{1}{2}}<\infty
$$

The space of functions $f \in L^{2}(D, H)$ which are analytic functions in $D$ (i.e., $\bar{\partial} f=0)$ is defined by

$$
A^{2}(D, H)=L^{2}(D, H) \cap \mathcal{O}(D, H)
$$

where $\mathcal{O}(D, H)$ denotes the Fréchet space of $H$-valued analytic functions on $D$ with respect to uniform topology. $A^{2}(D, H)$ is called the Bergman space for $D$. Note that $A^{2}(D, H)$ is a Hilbert space. The operator $T-z$ on the space $\mathcal{O}(D, H)$ has property $(\beta)$, which means by definition that $T-z$ is one-to-one and has closed range for every disc $D$.

Let us define now a Sobolev type space, called $W^{2}(D, H)$ where $D$ is a bounded disc in C. $W^{2}(D, H)$ will be the space of those functions $f \in L^{2}(D, H)$ whose derivatives $\bar{\partial} f, \bar{\partial}^{2} f$ in the sense of distributions still belong to $L^{2}(D, H)$. Endowed with the norm $\|f\|_{W^{2}}^{2}=\sum_{i=0}^{2}\left\|\bar{\partial}^{i} f\right\|_{2, D}^{2}, W^{2}(D, H)$ becomes a Hilbert space contained continuously in $L^{2}(D, H)$.

Now for $f \in C_{0}^{2}(\mathbf{C})$, let $M_{f}$ denote the operator on $W^{2}(D, H)$ given by multiplication by $f$. This has a spectral distribution of order 2 , defined by the functional calculus

$$
\Phi_{M}: C_{0}^{2}(\mathbf{C}) \longrightarrow \mathcal{L}\left(W^{2}(D, H)\right), \Phi_{M}(f)=M_{f}
$$

Therefore $M_{z}$ is a scalar operator of order 2 . In fact, it can be shown [Pu] that $M_{z}$ is subnormal.

\section{Subscalarity}

This section deals with the characterization for some $p$-hyponormal operators. Recall that an operator $T \in \mathcal{L}(H)$ is said to be $p$-hyponormal, $0<p \leq 1$, if $\left(T^{*} T\right)^{p} \geq\left(T T^{*}\right)^{p}$ where $T^{*}$ is the adjoint of $T$.

We need the following lemmas to give a proof of the main theorem.

Lemma 1 ([Xi], Lemma 2.1). Let $T=U|T|_{r}$ be the polar decomposition of $T, Q=$ $|T|_{r}-|T|_{l}, z=\rho e^{i \theta}, 0<\rho$, and $\left|e^{i \theta}\right|=1$ where $|T|_{r}=\left(T^{*} T\right)^{\frac{1}{2}}$ and $|T|_{l}=\left(T T^{*}\right)^{\frac{1}{2}}$. Then

$$
\|(T-z) f\|_{2, D}^{2}=\left\|\left(|T|_{r}-\rho\right) f\right\|_{2, D}^{2}+\rho\left\||T|_{r}^{\frac{1}{2}}\left(U-e^{i \theta}\right)^{*} f\right\|_{2, D}+\rho\langle Q f, f\rangle
$$

for all $f \in L^{2}(D, H)$.

For reference, we quote Lemma 2 from $[\mathrm{Pu}]$.

Lemma $2([\mathrm{Pu}]$, Proposition 2.1). For every bounded disk $D$ in $\mathbf{C}$ there is a constant $C_{D}$, such that for an arbitrary operator $T \in \mathcal{L}(H)$ and $f \in W^{2}(D, H)$ we have

$$
\|(I-P) f\|_{2, D} \leq C_{D}\left(\left\|(T-z)^{*} \bar{\partial} f\right\|_{2, D}+\left\|(T-z)^{*} \bar{\partial}^{2} f\right\|_{2, D}\right)
$$

where $P$ denotes the orthogonal projection of $L^{2}(D, H)$ onto the Bergman space $A^{2}(D, H)$. 
For $p$-hyponormal operator $T=U|T|$, Aluthge ([Al] $)$ introduced the operator $\tilde{T}=|T|_{r}^{\frac{1}{2}} U|T|_{r}^{\frac{1}{2}}$ and showed very interesting results on $\tilde{T}$.

Lemma $3([\mathrm{Al}])$. Let $T=U|T|_{r}$ be a p-hyponormal operator, $0<p<1$, and $U$ unitary. Then the operator $\tilde{T}=|T|_{r}^{\frac{1}{2}} U|T|_{r}^{\frac{1}{2}}$ is hyponormal if $\frac{1}{2} \leq p<1$, and $\left(p+\frac{1}{2}\right)$-hyponormal if $0<p<\frac{1}{2}$.

Lemma 4. Let $T=U|T|_{r}$ be semi-hyponormal and let $U$ be unitary. Let $D$ be a bounded disk which containes $\sigma(T)$. Then the map $V: H \rightarrow H(D)$ defined by $V h=\widetilde{1 \otimes h}\left(\equiv 1 \otimes h+\overline{(T-z) W^{2}(D, H)}\right)$ is one-to-one and has closed range, where $1 \otimes h$ denotes the constant function sending any $z \in D$ to $h$.

Proof. Let $h_{n} \in H$ and $f_{n} \in W^{2}(D, H)$ be sequences such that

$$
\lim _{n \rightarrow \infty}\left\|(T-z) f_{n}+1 \otimes h_{n}\right\|_{W^{2}}=0 .
$$

Then by the definition of the norm of Sobolev space (1) implies

$$
\lim _{n \rightarrow \infty}\left\|(T-z) \bar{\partial}^{i} f_{n}\right\|_{2, D}=0
$$

for $i=1,2$. Since $T$ is a semi-hyponormal operator, Lemma 1 and equation (2) imply

$$
\left\{\begin{array}{l}
\lim _{n \rightarrow \infty}\left\|\left(|T|_{r}-\rho\right) \bar{\partial}^{i} f_{n}\right\|_{2, D}=0, \\
\lim _{n \rightarrow \infty} \rho\left\||T|{ }_{r}^{\frac{1}{2}}\left(U-e^{i \theta}\right)^{*} \bar{\partial}^{i} f_{n}\right\|_{2, D}=0, \quad \text { and } \\
\lim _{n \rightarrow \infty} \rho\left\langle Q \bar{\partial}^{i} f_{n}, \bar{\partial}^{i} f_{n}\right\rangle=0
\end{array}\right.
$$

We note that for $i=1,2$

$$
\begin{aligned}
(T-z)^{*} \bar{\partial}^{i} f_{n}= & |T|_{r}^{\frac{1}{2}}\left[|T|_{r}^{\frac{1}{2}}\left(U-e^{i \theta}\right)^{*} \bar{\partial}^{i} f_{n}\right] \\
& +e^{-i \theta}\left[\left(|T|_{r}-\rho\right) \bar{\partial}^{i} f_{n}\right] .
\end{aligned}
$$

By equations (3) and (4), we get

$$
\lim _{n \rightarrow \infty}\left\|(T-z)^{*} \bar{\partial}^{i} f_{n}\right\|_{2, D}=0 .
$$

Lemma 2 and equation (5) imply

$$
\lim _{n \rightarrow \infty}\left\|(I-P) f_{n}\right\|_{2, D}=0,
$$

where $P$ denotes the orthogonal projection of $L^{2}(D, H)$ onto $A^{2}(D, H)$. Then by (1)

$$
\lim _{n \rightarrow \infty}\left\|(T-z) P f_{n}+1 \otimes h_{n}\right\|_{2, D}=0 .
$$

Let $\Gamma$ be a curve in $D$ surrounding $\sigma(T)$. Then for $z \in \Gamma$

$$
\lim _{n \rightarrow \infty}\left\|P f_{n}(z)+(T-z)^{-1}\left(1 \otimes h_{n}\right)\right\|=0
$$

uniformly. Hence

$$
\lim _{n \rightarrow \infty}\left\|\frac{1}{2 \pi i} \int_{\Gamma} P f_{n}(z) d z+h_{n}\right\|=0 .
$$

But by Cauchy's theorem,

$$
\int_{\Gamma} P f_{n}(z) d z=0 .
$$

Hence $\lim _{n \rightarrow \infty} h_{n}=0$. Thus $V$ is one-to-one and has closed range. 
Proposition 5. Let $T=U|T|_{r}$ be a p-hyponormal operator with the property $0 \notin$ $\sigma\left(|T|_{r}^{\frac{1}{2}}\right), 0<p<1$, and $U$ unitary. Let $D$ be a bounded disk which contains $\sigma(T)$. Then the map $V: H \rightarrow H(D)$ defined by $V h=\widetilde{1 \otimes h}\left(\equiv 1 \otimes h+\overline{(T-z) W^{2}(D, H)}\right)$ is one-to-one and has closed range, where $1 \otimes h$ denotes the constant function sending any $z \in D$ to $h$.

Proof. Let $h_{n} \in H$ and $f_{n} \in W^{2}(D, H)$ be sequences such that

$$
\lim _{n \rightarrow \infty}\left\|(T-z) f_{n}+1 \otimes h_{n}\right\|_{W^{2}}=0 .
$$

Then equation (7) implies

$$
\lim _{n \rightarrow \infty}\left\|(T-z) \bar{\partial}^{i} f_{n}\right\|_{2, D}=0
$$

for $i=1,2$.

(a) If $\frac{1}{2} \leq p<1$, then $T$ is semi-hyponormal. Therefore, Proposition 5 follows from Lemma 4.

(b) Let $0<p<\frac{1}{2}$. Since $T=U|T|_{r}$,

$$
\lim _{n \rightarrow \infty}\left\||T|_{r}^{\frac{1}{2}}\left(U|T|_{r}-z\right) \bar{\partial}^{i} f_{n}\right\|_{2, D}=0
$$

Since $\tilde{T}=|T|_{r}^{\frac{1}{2}} U|T|_{r}^{\frac{1}{2}}$, we have

$$
\lim _{n \rightarrow \infty}\left\|(\tilde{T}-z) \bar{\partial}^{i}\left(|T|_{r}^{\frac{1}{2}} f_{n}\right)\right\|_{2, D}=0 .
$$

Since $\tilde{T}$ is $\left(p+\frac{1}{2}\right)$-hyponormal by Lemma $3, \tilde{T}$ is semi-hyponormal. Let $\tilde{T}=W|\tilde{T}|_{r}$ be the polar decomposition. Lemma 1 and equation (9) imply

$$
\left\{\begin{array}{l}
\lim _{n \rightarrow \infty}\left\|\left(|\tilde{T}|_{r}-\rho\right) \bar{\partial}^{i}\left(|T|_{r}^{\frac{1}{2}} f_{n}\right)\right\|_{2, D}=0, \\
\lim _{n \rightarrow \infty} \rho\left\||\tilde{T}|_{r}^{\frac{1}{2}}\left(W-e^{i \theta}\right)^{*} \bar{\partial}^{i}\left(|T|_{r}^{\frac{1}{2}} f_{n}\right)\right\|_{2, D}=0, \quad \text { and } \\
\lim _{n \rightarrow \infty} \rho\left\langle Q \bar{\partial}^{i}\left(|T|_{r}^{\frac{1}{2}} f_{n}\right), \bar{\partial}^{i}\left(|T|_{r}^{\frac{1}{2}} f_{n}\right)\right\rangle=0 .
\end{array}\right.
$$

Now we note that for $i=1,2$

$$
\begin{aligned}
(\tilde{T}-z)^{*} \bar{\partial}^{i}\left(|T|_{r}^{\frac{1}{2}} f_{n}\right)= & |\tilde{T}|_{r}^{\frac{1}{2}}\left[|\tilde{T}|_{r}^{\frac{1}{2}}\left(W-e^{i \theta}\right)^{*} \bar{\partial}^{i}\left(|T|_{r}^{\frac{1}{2}} f_{n}\right)\right] \\
& +e^{-i \theta}\left[\left(|\tilde{T}|_{r}-\rho\right) \bar{\partial}^{i}\left(|T|_{r}^{\frac{1}{2}} f_{n}\right)\right] .
\end{aligned}
$$

By (10) and (11), we get

$$
\lim _{n \rightarrow \infty}\left\|(\tilde{T}-z)^{*} \bar{\partial}^{i}\left(|T|_{r}^{\frac{1}{2}} f_{n}\right)\right\|_{2, D}=0 .
$$

Lemma 2 and equation (12) imply

$$
\lim _{n \rightarrow \infty}\left\|(I-P)|T|_{r}^{\frac{1}{2}} f_{n}\right\|_{2, D}=0 .
$$

Since $|T|_{r}^{\frac{1}{2}}(T-z)=(\tilde{T}-z)|T|_{r}^{\frac{1}{2}}$ and $0 \notin \sigma\left(|T|_{r}^{\frac{1}{2}}\right)$, it follows from $(7)$ that $\sigma(T)=$ $\sigma(\tilde{T})$ and

$$
\lim _{n \rightarrow \infty}\left\|(\tilde{T}-z)|T|_{r}^{\frac{1}{2}} f_{n}+|T|_{r}^{\frac{1}{2}}\left(1 \otimes h_{n}\right)\right\|_{2, D}=0 .
$$

By (13) and (14), we have

$$
\lim _{n \rightarrow \infty}\left\|(\tilde{T}-z) P\left(|T|_{r}^{\frac{1}{2}} f_{n}\right)+|T|_{r}^{\frac{1}{2}}\left(1 \otimes h_{n}\right)\right\|_{2, D}=0 .
$$


Let $\Gamma$ be a curve in $D$ surrounding $\sigma(T)(=\sigma(\tilde{T}))$. Then for $z \in \Gamma$

$$
\lim _{n \rightarrow \infty}\left\|P\left(|T|_{r}^{\frac{1}{2}} f_{n}(z)\right)+(\tilde{T}-z)^{-1}\left(|T|_{r}^{\frac{1}{2}}\left(1 \otimes h_{n}\right)\right)\right\|=0
$$

uniformly. Hence

$$
\lim _{n \rightarrow \infty}\left\|\frac{1}{2 \pi i} \int_{\Gamma} P\left(|T|_{r}^{\frac{1}{2}} f_{n}(z)\right) d z+|T|_{r}^{\frac{1}{2}} h_{n}\right\|=0 .
$$

But by Cauchy's theorem,

$$
\frac{1}{2 \pi i} \int_{\Gamma} P\left(|T|_{r}^{\frac{1}{2}} f_{n}(z)\right) d z=0
$$

Therefore $\lim _{n \rightarrow \infty}|T|_{r}^{\frac{1}{2}} h_{n}=0$. Since $0 \notin \sigma\left(|T|_{r}^{\frac{1}{2}}\right),|T|_{r}^{\frac{1}{2}}$ is bounded below. Hence $\lim _{n \rightarrow \infty} h_{n}=0$.

Theorem 6. Let $T=U|T|_{r}$ be p-hyponormal, $0<p<1$, and $U$ unitary. If $0 \notin \sigma\left(|T|_{r}^{\frac{1}{2}}\right)$, then $T$ is subscalar of order 2 .

Proof. Consider an arbitrary bounded open disk $D$ in the complex plane $\mathbf{C}$ and the quotient space

$$
H(D)=W^{2}(D, H) / \overline{(T-z) W^{2}(D, H)}
$$

endowed with the Hilbert space norm. The class of a vector $f$ or an operator on $H(D)$ will be denoted by $\tilde{f}$, respectively $\tilde{A}$. Let $M$ be the operator of multiplication by $z$ on $W^{2}(D, H)$. As noted at the end of section $2, M$ is a scalar of order 2 and has a spectral distribution $\Phi$. Let $S \equiv \tilde{M}$. Since $\overline{(T-z) W^{2}(D, H)}$ is invariant under every operator $M_{f}, f \in C^{2}(D)$, we infer that $S$ is a scalar operator of order 2 with spectral distribution $\tilde{\Phi}$.

Consider the natural map $V: H \longrightarrow H(D)$ defined by $V h=\widetilde{1 \otimes h}$, for $h \in H$, where $1 \otimes h$ denotes the constant function identically equal to $h$. Note that $V T=$ $S V$. In particular ran $V$ is an invariant subspace for $S$. Since $V$ is one-to-one and has closed range by Proposition 5, $T$ is subscalar of order 2 .

Corollary 7. Every invertible p-hyponormal operator is subscalar of order 2.

Proof. Assume $T=U|T|_{r}$ is an invertible $p$-hyponormal operator where $U$ is unitary. Then $|T|_{r}$ is invertible. By [Ru, Theorem 12.33], $|T|_{r}^{\frac{1}{2}}$ is invertible. Therefore, $0 \notin \sigma\left(|T|_{r}^{\frac{1}{2}}\right)$. By Theorem $6, T$ is subscalar of order 2 .

Corollary 8. Let $T=U|T|_{r}$ be a p-hyponormal operator with the property $0 \notin$ $\sigma\left(|T|_{r}^{\frac{1}{2}}\right), 0<p<1$, and $U$ unitary. If $\sigma(T)$ has interior in the plane, then $T$ has a non-trivial invariant subspace.

Proof. The corollary follows from Theorem 6 and [Es].

Corollary 9. Let $T$ be as in Corollary 8. Then $T$ has the property $(\beta)$.

Proof. Since every subscalar operator has the property $(\beta)$, the corollary follows from Theorem 6 .

Recall that an $X$ in $\mathcal{L}(H, K)$ is called a quasi-affinity if it has trivial kernel and dense range. An operator $A$ in $\mathcal{L}(H)$ is said to be a quasi-affine transform of an operator $T$ in $\mathcal{L}(K)$ if there is a quasi-affinity $X$ in $\mathcal{L}(H, K)$ such that $X A=T X$ (notation: $A \prec T$ ). 
Corollary 10. Let $T$ be as in Corollary 8. If $A$ is any operator such that $A \prec T$, then $\sigma(T) \subseteq \sigma(A)$.

Proof. This is clear from [Ko, Theorem 3.2] and Corollary 9.

Corollary 11. Under the same hypothesis as Corollary 10, $A \in \mathcal{L}(H)$ is quasisubscalar.

Proof. Let $X \in \mathcal{L}(H, K)$ be a quasi-affinity such that $X A=T X$. Since $V$ (in the construction of $V$ and $S$ ) and $X$ are one-to-one, $V X$ is one-to-one. Therefore $V X$ implements the quasi-subscalar properties. Thus $A$ is quasi-subscalar.

\section{REFERENCES}

[Al] A. Aluthge, On p-hyponormal operators for $0<p<1$, Int. Eq. and Op. Th. 13(1990), 307-315. MR 91a:47025

[Es] J. Eschmeier, Invariant subspaces for subscalar operators, Arch. Math. 52(1989), 562-570. MR 90h:47016

[Ko] E. Ko, On a Clary theorem, Bull.Kor.Math.Soc. 33(1996), 29-33. MR 97e:47031

[MP] M. Martin and M. Putinar, Lectures on Hyponormal Operators, Birkhäuser Verlag, Boston, Vol. 39, 1989. MR 91c:47041

$[\mathrm{Pu}]$ M. Putinar, Hyponormal operators are subscalar, J. Op. Th. 12(1984), 385-395. MR 85h:47027

[Ru] W. Rudin, Functional Analysis, McGraw-Hill Int. Ed., 2nd Ed., 1991. MR 92k:46001

[Xi] D. Xia, Spectral Theory of Hyponormal Operators, Birkhäuser Verlag, Boston, 1983. MR $\mathbf{8 7 j}: 47036$

Department of Mathematics, Ewha Women's University, Seoul 120-750, Korea

E-mail address: eiko@mm.ewha.ac.kr 